\title{
High-speed photometry of the pre-cataclysmic binary HW Virginis and its orbital period change
}

\author{
C. İbanoğlu, Ö. Çakırlı, G. Taş, and S. Evren
}

Ege University Observatory, 35100 Bornova, İzmir, Turkey

Received 26 June 2003 / Accepted 8 August 2003

\begin{abstract}
The broad band $B$ and $V$ light curves of the pre-cataclysmic eclipsing binary HW Vir were obtained. All the available eclipse timings, including the new ones, spanning 19 years were analyzed under the third-body hypothesis. The residuals between the observed and calculated times of mid-eclipse show a long-term sinusoidal variation. The analysis yields the parameters of the third-body orbit, as well as limiting mass for the tertiary object. The result of this analysis gives a light-time semi-amplitude of $112 \mathrm{~s}$, an orbital period of $18.8 \mathrm{yr}$ and an eccentricity of 0.12 . The mass of the third star is below the theoretical threshold of $0.07 M_{\odot}$ for a hydrogen burning star. Its minimum mass is about $0.022 M_{\odot}$ and for a wide range of inclinations of third-body orbit, i.e. $i \geq 19^{\circ}$, the mass is smaller than $0.07 M_{\odot}$. Therefore, we suggest that the third star may be a brown dwarf candidate. Combining the semi-amplitudes of the radial velocities and the photometric light curve solution has allowed us to model the short-period detached binary HW Vir. The luminosity and radius of the dM companion are slightly larger than that given by low-mass models.
\end{abstract}

Key words. stars: individual: HW Vir - stars: binaries: eclipsing - techniques: photometry

\section{Introduction}

The studies made by Maxted et al. (2002) and Saffer et al. (2001) have revealed that many sdB stars are members of close binary systems. Therefore, the eclipsing systems that contain sdB stars are crucial both for determination of their masses and evolution. However, there are only three eclipsing pairs known to include a sdB companion. These are HW Vir, PG 1336-018 and HS 0705+6700 discovered, recently, by Drechsel et al. (2001).

HW Vir was discovered as an eclipsing binary by Menzies (1986). The first light curve published by Menzies \& Marang (1986) includes both primary and secondary eclipses but the light curve displays a striking reflection effect. The light curve analyses of the system were made by different investigators, adopting different temperatures for the main sequence component (Menzies \& Marang 1986; Wood et al. 1993; Çakirli \& Devlen 1999). The temperature of the sdB companion was estimated in a wide range between 26000 and $36000 \mathrm{~K}$. Later Wood \& Saffer (1999) derived the atmospheric parameters of the sdB star using the spectra taken at either side of the primary eclipse, where the contribution from the secondary star flux is negligible. They found the temperature, gravity and helium

Send offprint requests to: $\mathrm{C}$. İbanoğlu, e-mail: ibanoglu@astronomy.sci.ege.edu.tr abundance as $T_{\mathrm{e}}=28488 \mathrm{~K}, \log g=5.63$ and $N(\mathrm{He}) / N(\mathrm{H})=$ 0.0066 , respectively. The low helium abundance points out the compact object in HW Vir is a normal classical sdB star.

The strong orbital period decrease in the sdB eclipsing binary HW Vir has been noted by Kilkenny et al. (1994). They have analyzed the eclipse timings over a 9-year baseline and concluded that the most favorable mechanism for the strong orbital period decrease is a possible angular momentum loss via magnetic breaking in a modest stellar wind. However, they did not rule out a third body in the system as a possible mechanism for the orbital period change but this hypothesis should be observationally testable with observations to be made later. The eclipse timings obtained between 1984 and 1999 were re-analyzed by Çakirli \& Devlen (1999) assuming most plausible mechanism of the orbital period change is the light-time effect. They suggested the period of revolution to be about 19 years around the third body. Later on Wood \& Saffer (1999), Kilkenny et al. (2000), Kiss et al. (2000) and very recently Kilkenny et al. (2003) discussed the O-C diagram for HW Vir. Kilkenny et al. (2003) found that the available data indicate that the third body is likely to be a brown dwarf unless the inclination of the outer orbit is $i<20$ degrees.

We have continued photometric observations of this precataclysmic system: $i$ ) to clarify the most acceptable cause of the orbital period change, ii) to redetermine the orbital and physical parameters of the system using a good light curve obtained with high accuracy. 


\section{Observations and light curves}

We observed HW Vir during the 2001, 2002 and 2003 observing seasons. Many light curves of the system in Johnson's wide-band $B$ and $V$ filters were obtained with a high-speed three-channel photon-counting photometer HSTCP attached to the $48 \mathrm{~cm}$ Cassegrain telescope at Ege University Observatory. Such photometers, like HSTCP designed for the time-series multicolor photometry of rapidly variable stars, are indispensable for investigation of rapid phenomena, especially in variable stars. The monitoring was continuous for about three minutes, except for interruptions about once every three minutes to check the comparison star on channel two (target star on channel one, sky-background on channel three). All three channels of the photometer coincide with the telescope's main axis. This photometer allows us to observe target star, comparison and sky background simultaneously in each filter. For a brief description of the equipment or detailed techniqual information please see Kalytis (1999).

In order to have a high counting rate a ten-second integration time was chosen for each measurement and we used a fancooling system in front of each Hamamatsu R1463P multiplier. The main comparison star was HD 110647 (BD -8³411), as used by Çakirli \& Devlen (1999). The extinction coefficients were obtained using the observations of the comparison star. Then, all differential magnitudes, in the sense variable minus comparison, were corrected for differential atmospheric extinction. The times were also reduced to the Sun's center. The orbital phases were calculated using the light elements given by Çakirli \& Devlen (1999) as

$$
\text { HJD }(\text { Min I })=2445730.5565+0.116719582 \times \mathrm{E} .
$$

Our observations enabled us to determine 10 times of primary and 8 secondary minima of HW Vir in two passbands. The times of minima were determined separately for both filters using the method of Kwee \& van Woerden (1956). These times of minima are presented in Table 1 with their standard deviations. The photoelectric light curves obtained in 2002 are depicted in Fig. 1. During each observing night the star was observed for more than four hours which covers one and half of the orbital period. As seen from Fig. 1, high-speed observations of the system do not show any change in the brightness over short time intervals. The light curve of the system is stable during a three-year time interval and high-speed observations allow us to better define the contact times and also to determine the eclipse timings.

\section{Period variation and its most probable cause}

The evolutionary status of HW Vir has been discussed in detail by Wood et al. (1993). They suggest that mass transfer from the main sequence star to the compact object will begin when the normal star fills its corresponding Roche lobe. To bring into contact configuration the orbital period should be decreased by angular momentum loss due to gravitational radiation. Their calculation indicates that when the orbital period decreases to $109 \mathrm{~min}$ the main-sequence component will be in contact with the Roche lobe for a mass ratio of 0.3 and

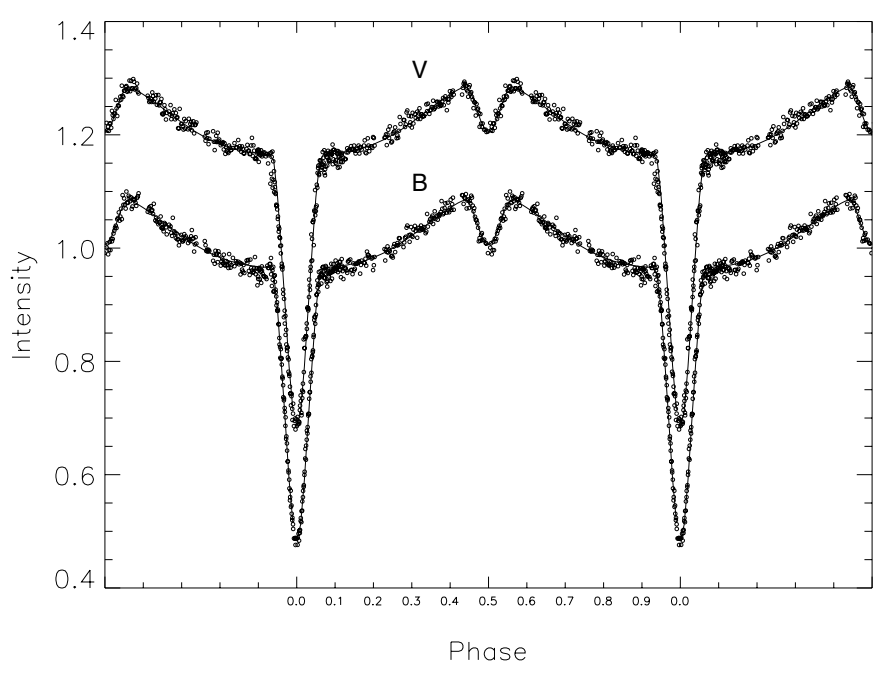

Fig. 1. $B$ and $V$ light curves of HW Vir obtained on March 14, 2002. The solid lines represent the best fit solution according to the parameters listed in Table 4 . The $V$-light curve was shifted 0.25 on the ordinate.

Table 1. New primary and secondary eclipse timings for HW Virginis.

\begin{tabular}{|c|c|c|c|c|c|}
\hline $\begin{array}{l}\text { JD } \quad \text { (Hel.) } \\
2400000+\end{array}$ & Type & Filter & $\sigma$ & $E$ & $\mathrm{O}-\mathrm{C}$ \\
\hline 52348.43644 & $I$ & $B$ & .00081 & 56699 & -0.00364 \\
\hline .43651 & $I$ & $V$ & .00064 & 56699 & -0.00357 \\
\hline .49495 & $I I$ & $B$ & .00111 & 56699.5 & -0.00349 \\
\hline .49505 & $I I$ & $V$ & .00100 & 56699.5 & -0.00339 \\
\hline .55319 & $I$ & $V$ & .00091 & 56700 & -0.00361 \\
\hline .55329 & $I$ & $B$ & .00089 & 56700 & -0.00351 \\
\hline 52373.29764 & $I$ & $B$ & .00077 & 56912 & -0.00371 \\
\hline .29775 & $I$ & $V$ & .00068 & 56912 & -0.00360 \\
\hline .35617 & $I I$ & $B$ & .00095 & 56912.5 & -0.00354 \\
\hline .35624 & $I I$ & $V$ & .00093 & 56912.5 & -0.00347 \\
\hline .41431 & $I$ & $V$ & .00088 & 56913 & -0.00376 \\
\hline .41435 & $I$ & $B$ & .00086 & 56913 & -0.00372 \\
\hline 52756.31274 & $I I$ & $B$ & .00041 & 60193.5 & -0.00392 \\
\hline .31414 & $I I$ & $V$ & .00029 & 60193.5 & -0.00252 \\
\hline .37116 & $I$ & $B$ & .00009 & 60194 & -0.00386 \\
\hline .37123 & $I$ & $V$ & .00003 & 60194 & -0.00379 \\
\hline .42936 & II & $B$ & .00011 & 60194.5 & -0.00402 \\
\hline .42969 & $I I$ & $V$ & .00006 & 60194.5 & -0.00369 \\
\hline
\end{tabular}

the system will be seen as a cataclysmic variable. Therefore, the variation in the orbtial period of HW Vir is important from this point of view, i.e., creation of a cataclysmic system.

Kilkenny et al. (1994) studied the orbital period change for the first time and concluded that the period of HW Vir was decreasing. They have discussed possible mechanisms for the period decrease but they could not reach a conclusion as to which one was the most likely candidate. The only two possible 
mechanisms, i.e. a third body in the system or magnetic braking in a weak stellar wind, were suggested as the most plausible cause for the period change. Later on Wood \& Saffer (1999), Kilkenny et al. (2000) and Kiss et al. (2000) re-analyzed all the $\mathrm{O}-\mathrm{C}$ residuals available. The former and the latter represented the residuals by two separate linear fits. These studies indicate that the orbital period of the system changed abruptly around 1991. On the other hand, Kilkenny et al. (2000) found that HW Vir shows a clear and substantial period decrease over 16 years. The eclipse timings were fitted with polynomials of up to sixth order. They found that the first three polynomial fits to the eclipse timings - i.e. linear, quadratic and cubic - seem to be insufficient. They tried to use a 6th-order polynomial to achieve a good fit but this attempt failed and the residuals showed systematic deviations. Therefore, they concluded that the observed period change for HW Vir may be produced by either orbiting of the binary system around a third companion or mass loss from the system as weak stellar wind. Recently, Kilkenny et al. (2003) analyzed all the available timings over the past seventeen years. Although the cause of distinct period change has not been obvious they conclude that a clear reversal from decreasing to increasing period may be taken as indicative of the third body in the system.

We collected all the available timings of both primary and secondary eclipses published by different authors and added the new ones given in Table 1. The typical errors of the new times of the mid-eclipse are less than about $0.0009 \mathrm{~d}$ for the primary eclipse, but a little larger for the shallower secondary eclipse. The $\mathrm{O}-\mathrm{C}$ residuals were obtained by using the ephemeris given by Çakirli \& Devlen (1999). These residuals seem to show a sine-like change rather than linear or parabolic changes. Sinusoidal variation in the orbital period of an eclipsing binary may be attributable either to an orbital motion around a third-body or to an apsidal motion. Since the orbit of HW Vir is circular, revealed by both the light and radial velocity curves, and any shift of the mid-secondary eclipse with respect to the mid-primary was detected, one may easily rule out apsidal motion as a possible cause of period change. Therefore, the mechanism remaining is the possibility of orbiting the eclipsing binary around a third body. For this reason, we analyzed all the available times of mid-primary minimum under an assumption of a third body and used the conventional formulae given by İbanoğlu et al. (2000) and references therein. Since the secondary eclipse is shallow, the times of mid-secondary eclipses have larger uncertainties. Therefore, the $\mathrm{O}-\mathrm{C}$ residuals for the secondary eclipse were excluded from the analysis. By applying a least squares solution we obtained the parameters for the third-body orbit, listed in Table 2 with their standard deviations.

In this table, $A$ is the semi-amplitude of the light-time effect and $a \sin i$ is the projected semi-major axis of the relative orbit of the eclipsing pair around the common center-of-mass. $i, e$ and $\omega$ are the inclination, eccentricity and longitude of the periastron of the third-body orbit, respectively. $T_{3}$ and $P_{3}$ are the time of periastron passage and the third-body period. $T_{0}$ and $P$ are the light elements of the eclipsing pair. The light-time effect due to orbit of the eclipsing pair around the third body has been computed with the parameters given in Table 2 and
Table 2. Orbital solution for the third component of HW Vir.

\begin{tabular}{lcc}
\hline \hline Parameter & Value & $\sigma$ \\
\hline$T_{0}(\mathrm{HJD})$ & 45730.55743 & 0.00005 \\
$P_{0}($ day $)$ & 0.1167195 & $4 \times 10^{-7}$ \\
$a_{12} \sin i(\mathrm{~km})$ & $3.05 \times 10^{7}$ & $0.50 \times 10^{7}$ \\
$e$ & 0.12 & 0.02 \\
$\omega(\mathrm{deg})$ & 49 & 2 \\
$T_{3}(\mathrm{HJD})$ & 48112 & 119 \\
$P_{3}(\mathrm{yr})$ & 18.8 & 0.4 \\
$A(\mathrm{day})$ & 0.0013 & 0.0001 \\
$f(m)\left(M_{\odot}\right)$ & $2.40 \times 0^{-5}$ & $1.08 \times 10^{-5}$ \\
$\Sigma(\mathrm{O}-\mathrm{C})^{2}$ & 0.00018 & \\
\hline
\end{tabular}

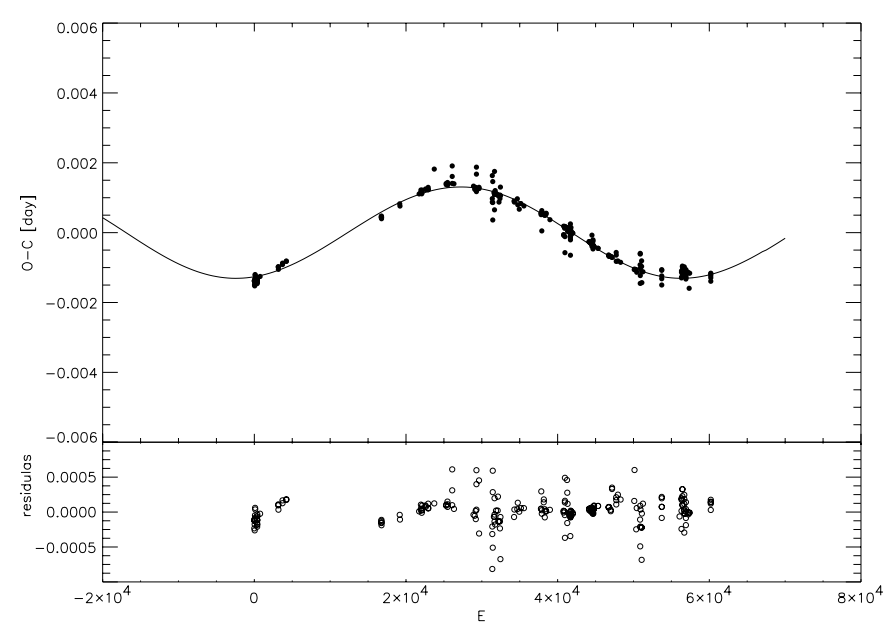

Fig. 2. Plot of the residuals of the eclipse timings using a linear ephemeris. The best-fitting curve assuming a third-body perturbation is shown as a solid line (upper panel). Residuals after subtracting the third-body perturbation are also shown in the lower panel.

subtracted from each time of minimum. The remaining residuals are shown in the lower panel of Fig. 2 .

The period of the revolution of the eclipsing pair around the center-of-mass with the third body is $18.8 \mathrm{yr}$ and the time span following the discovery of the eclipsing nature of HW Vir covers more than $100 \%$ of the period predicted in this study.

The $\mathrm{O}-\mathrm{C}$ analysis allows us to determine the mass function of the unseen component and to estimate its mass by

$f(m)=\frac{\left(a_{12} \sin i\right)^{3}}{P_{3}^{2}}=\frac{\left(m_{3} \sin i\right)^{3}}{\left(m_{12}+m_{3}\right)^{2}}$

where $m_{3}$ and $f(m)$ are the mass and mass function of the thirdbody and $m_{12}$ is the total mass of the eclipsing pair. We calculated the mass function to be $2.40( \pm 1.08) \times 10^{-5}$ solar mass using the $a_{12} \sin i$ of $0.204 \mathrm{AU}$ and a period of $18.8 \mathrm{yr}$. The masses of the components of the eclipsing pair were recently derived by Wood \& Saffer (1999) as 0.48 and 0.14 solar masses. If the eclipsing pair is orbiting around the common center-of-mass with a period of $18.8 \mathrm{yr}$ one may easily estimate the mass of 
Table 3. The normalized Fourier $\left(l=1.0\right.$ for $\left.\theta=90^{\circ}\right)$ coefficients.

\begin{tabular}{rrrrrr}
\hline \hline Filter & \multicolumn{1}{c}{$A_{0}$} & \multicolumn{1}{c}{$A_{1}$} & $A_{2}$ & $B_{1}$ & \multicolumn{1}{c}{$B_{2}$} \\
\hline$B$ & 1.0164 & -0.0848 & 0.0186 & 0.0021 & -0.0015 \\
& \pm 6 & \pm 9 & \pm 10 & \pm 6 & \pm 6 \\
$V$ & 1.0205 & -0.0983 & 0.0206 & 0.0001 & 0.0006 \\
& \pm 5 & \pm 118 & \pm 138 & \pm 5 & \pm 5 \\
\hline
\end{tabular}

the tertiary body from the mass function. Its mass, of course, depends on the inclination of the eclipsing pairs' orbit. For the inclination of $90^{\circ}, 60^{\circ}$ and $30^{\circ}$ we calculate the third body's mass to be $0.022,0.025$ and $0.044 M_{\odot}$. If this body is really a star, i.e. its mass should be greater than $0.07 M_{\odot}$, the limiting mass for stable hydrogen burning, the orbital inclination of the long period orbit should be smaller than $19^{\circ}$. The mass of the tertiary component is of the order of a brown dwarfs' mass for a very wide range of inclination. As pointed out by Kirkpatric et al. (2000) more than hundred brown dwarf candidates have been detected from the infrared surveys. Although a number of different models have been published so far to explain their observed properties, the dynamical masses could be derived only for a few brown dwarfs dynamically bound to a star. The most well-known binary companion brown dwarf is Gliese 229B, which has been studied by many observers. The second multiple stars' brown dwarf member was recently suspected by Guinan \& Ribas (2001) in V471 Tau, a white-dwarf and red-dwarf eclipsing binary. Eclipsing binaries showing a light-time effect present a possibility for detecting such low mass companions, in addition to direct imaging and radial velocity techniques.

As reported by Chamblis (1992) and recently by Demircan (2000), a significant number of eclipsing binaries occur as components of triple star systems or higher-order multiple-star systems. A definite but not cyclic orbital period variation of an eclipsing binary can be regarded as evidence for the existence of a third body. The orbital periods of such binaries are very sensitive to the existence of a companion and are easily detectable from the $\mathrm{O}-\mathrm{C}$ analysis, unless the common center-ofmass is too close to the mass center of the eclipsing pair.

\section{Light curve analysis}

As shown in Fig. 1, the most striking feature of the light curve is the reflection as expected for a hot subdwarf plus cool lowmass eclipsing binary. We made a Fourier analysis of the light curve outside of the eclipses to estimate the reflection rate by the cool companion. The coefficients of the truncated Fourier series are given in Table 3.

The $A_{1}$ and $A_{2}$ terms are the contribution to the reflection effect. The latter coefficient is partially affected by the oblateness and the effect of tidal distortion on the light curves. The $B_{1}$ and $B_{2}$ terms indicate a difference between the maxima of the light curve and other perturbations, such as mass transfer, dark spots on one or two components etc. The $B$-coefficients are too small when compared with the others, therefore the light curve of HW Vir seems to be unaffected by other effects, such as the reflection effect.

By using the Fourier coefficients we obtained the brightness change from mid-primary to mid-secondary due to reflection effects of $0^{\mathrm{m}} 178$ in $B$ and 0.206 in the $V$ band. These values are in good agreement with those expected from the formula given by Maxted et al. (2002). The reflection rate for HW Vir gets stronger as we go to longer wavelengths. We compute the disk-averaged temperature of the cool main-sequence star to be 9000 and $10000 \mathrm{~K}$ for $B$ and $V$-band light curves, respectively. The temperatures for the illuminated hemisphere of the cool companion that we estimate from the reflection effect in $B$ and $V$ bands are a little smaller than that found by Kiss et al. (2000) and in good agreement with the value of $10800 \mathrm{~K}$ computed from the formula given by Maxted et al. (2002).

We have analyzed the $B$ and $V$ light curves separately by utilizing the Wilson and Devinney light curve synthesis code (Wilson \& Devinney 1971; Wilson 1998). First, we adjusted the geometrical parameters and the effective temperature of the hot primary. The minimum squared sum of residuals between observed and calculated luminosities was obtained for a temperature of $28700 \pm 200 \mathrm{~K}$ for the sdB star. This estimate depends on the colour change during the primary eclipse and is close to those obtained by Wood \& Saffer. Since Wood \& Saffer's estimation depends on the optical spectroscopy, which is more sensitive to the temperature, we adopt a temperature of $28500 \mathrm{~K}$ for the sdB component. After the first analysis we adjusted the bolometric albedo for the cooler companion. The effective temperature of the cool companion was found to be $3378 \pm 91 \mathrm{~K}$ for $B$ and $3129 \pm 199 \mathrm{~K}$ for $V$ band. The temperature estimated from the $V$ light curve analysis is in accordance with the mainsequence models for low-mass stars published by Dorman et al. (1989). However, the temperature found for the $B$ light curve is somewhat greater than that of models. It is clear that the unirradiated face of such a cool star will contribute negligible flux to the total flux when compared with the contribution of a hot subdwarf. We obtained the bolometric albedo for this star to be $0.73 \pm 0.04$ in $B$ and $0.98 \pm 0.03$ in $V$-band light curves. This estimation clearly indicates that the heated secondary component differs from the convective, low mass cool main-sequence stars. Therefore, it was decided to treat the limb darkening coefficients for this heated cool star as adjustable parameter. The analysis indicates that the limb darkening coefficients for this star are $0.73 \pm 0.03$ in $B$ and $0.43 \pm 0.01$ in $V$-band. The coefficients are substantially reduced, as expected.

Table 4 gives a list of the fixed and adjusted parameters. The light curves computed with these parameters are compared with the observations in Fig. 1. This figure clearly shows that the parameters found by light curve analysis represent very well the observed light curve of the system. The pronounced reflection effect is fitted very well.

We determined the fractional radii for the hot and cool components as $R_{1} / a=0.2307 \pm 0.0011, R_{2} / a=0.2122 \pm 0.0011$ respectively, where $a$ is the separation between the mass centers of the binary stars. Using the $K_{1}=82.3 \mathrm{~km} \mathrm{~s}^{-1}$, orbital inclination of 81.07 , and the mass ratio of 0.29 we derive $a=$ $0.853 R_{\odot}$, and $R_{1}=0.197 R_{\odot}$ and $R_{2}=0.181 R_{\odot}$. The radius 
Table 4. The parameters obtained from the $B, V$ light curves of HW Vir.

\begin{tabular}{|c|c|c|}
\hline $\begin{array}{l}\text { Fixed } \\
\text { parameters }\end{array}$ & $B$ & $V$ \\
\hline$T_{1}(\mathrm{~K})$ & 28500 & 28500 \\
\hline$A_{1}$ & 1.0 & 1.0 \\
\hline$g_{1}$ & 1.0 & 1.0 \\
\hline$g_{2}$ & 0.32 & 0.32 \\
\hline$x_{1}$ & 0.266 & 0.212 \\
\hline$q$ & 0.29 & 0.29 \\
\hline $\begin{array}{l}\text { Adjusted } \\
\text { parameters }\end{array}$ & $B$ & $V$ \\
\hline$i\left(^{\circ}\right)$ & $81.16 \pm 0.10$ & $80.97 \pm 0.10$ \\
\hline$\Omega_{1}$ & $4.7113 \pm 0.0105$ & $4.5849 \pm 0.0109$ \\
\hline$\Omega_{2}$ & $2.7936 \pm 0.0111$ & $2.6912 \pm 0.02143$ \\
\hline$T_{2}(\mathrm{~K})$ & $3378 \pm 91$ & $3129 \pm 199$ \\
\hline$L_{1}$ & $0.9998 \pm 0.0011$ & $0.9997 \pm 0.0011$ \\
\hline$L_{2}$ & 0.0002 & 0.0003 \\
\hline$A_{2}$ & $0.73 \pm 0.05$ & $0.98 \pm 0.03$ \\
\hline$x_{2}$ & $0.73 \pm 0.07$ & $0.43 \pm 0.03$ \\
\hline$r_{1}($ pole $)$ & $0.2258 \pm 0.0010$ & $0.2325 \pm 0.0011$ \\
\hline$r_{1}$ (point) & $0.2389 \pm 0.0011$ & $0.2360 \pm 0.0011$ \\
\hline$r_{1}($ side $)$ & $0.2275 \pm 0.0012$ & $0.2344 \pm 0.0011$ \\
\hline$r_{1}$ (back) & $0.2286 \pm 0.0011$ & $0.2356 \pm 0.0011$ \\
\hline$r_{2}($ pole $)$ & $0.1989 \pm 0.0011$ & $0.2135 \pm 0.0011$ \\
\hline$r_{2}$ (point) & $0.2148 \pm 0.0011$ & $0.2363 \pm 0.0011$ \\
\hline$r_{2}($ side $)$ & $0.2025 \pm 0.0011$ & $0.2183 \pm 0.0011$ \\
\hline$r_{2}$ (back) & $0.2112 \pm 0.0011$ & $0.2304 \pm 0.0011$ \\
\hline
\end{tabular}

of the primary component determined in this study is $12 \%$ greater than found by Wood \& Saffer (1999). In contrast to Wood \& Saffer, the cool, less massive companion is slightly smaller than the hot component and our solution is in agreement with that of Wlodarczyk \& Olszewski (1994). The lowmass cool companion is about $9 \%$ oversized for its mass relative to the Dorman et al. model. The M-dwarf seems to be more luminous in the mass-luminosity plane. Two posibble reasons for the unusually large radius of the $\mathrm{dM}$ star may be either $i$ ) irradiation of the $\mathrm{dM}$ star by the sdB component, or, ii) the $\mathrm{dM}$ companion is so covered by starspots that convective energy transport is inhibited, causing the radius to increase. However, there are no clues about the magnetic activity of cool companion; therefore, the former may be an appropriate explanation, because not only the radius but also the luminosity of this star is larger than the value expected from models.

\section{Conclusions}

The eclipsing binary HW Vir, consisting of a hot $\mathrm{sdB}$ and a cool main-sequence star, was observed using a high-speed three-channel photometer. Many precise light curves and times of mid-primary and mid-secondary eclipses were obtained. The period change of the system and plausible causes were reexamined. The last times of mid-eclipses revealed that the instantenous period of the system indicates a sine-like change over the past 19 years. The residuals from the linear fit are clearly asymmetric which implies that a quadratic fit is inappropriate. Therefore, we analyzed all the residuals obtained to date under the assumption of a third body hypothesis. Using a least squares solution we obtained parameters for the thirdbody orbit. The modeling yields an orbital period for the thirdbody of $P_{3}=18.8 \pm 0.4 \mathrm{yr}$, a light-time semi-amplitude of $112 \mathrm{~s}, a_{12} \sin i=0.204 \pm 0.033 \mathrm{AU}$ and an eccentricity of $e_{3}=0.12$. Adopting a total mass for the eclipsing binary of $0.62 M_{\odot}$, we computed the mass function for the triple system as $f(m)=2.40( \pm 1.08) \times 10^{-5} M_{\odot}$. The mass of the third-body is $M_{3} \sin i_{3} \simeq 0.022$ and its orbital semi-major axis is $a_{3} \simeq 6.1 \mathrm{AU}$. As long as the inclination of the orbital plane of the third body is $i \geq 19^{\circ}$, the mass of this object is below the theoretical threshold of $\sim 0.07 M_{\odot}$ for a hydrogen burning star. The minimum mass of the third component was found to be $0.022 M_{\odot}$ which would be too massive to be a planet. Therefore, the third component in the HW Vir system is a possible candidate for a brown dwarf star.

The light curves obtained using HSTCP were analyzed for the parameters of the eclipsing binary orbit and as well as for the physical properties of the components. We have carried out an analysis of the outside eclipse light curve and determined disk-averaged temperatures for the cool companion facing the hot sdB star. The effective temperature of the low-mass cool companion is in accord with the theoretical models. However, its luminosity and radius are slightly larger than the values predicted from the models. These differences were attributed to either heating of the cool star by radiant energy coming from the sdB star, or large spots on its surface.

Acknowledgements. We would like to express our sincere thanks to the personnel of the Ege University Observatory for their valuable aid during the installation of the new photometer on the telescope. We would also like to thank the reviewer, Dr. P. F. L. Maxted, for his timely review and helpful comments.

\section{References}

Chambliss, C. R. 1992, PASP, 104, 663

Çakirli, Ö., \& Devlen, A. 1999, A\&A, 136, 27

Demircan, O. 2000, in Variable Stars As Essential Astrophysical Tools, ed. C. İbanoglu (Kluwer Academic Publishers), 615

Drechsel, H., Heber, U., \& Napiwotzski, R. 2001, A\&A, 379, 893

Dorman, B., Nelson, L., \& Chau, W. Y. 1989, ApJ, 342, 1003

Guinan, E. F., \& Ribas, I. 2001, ApJ, 546, L43

İbanoğlu, C., Çakirli, Ö., Değirmenci, Ö., et al. 2000, A\&A, 354, 188 Kalytis, R. 1999, Tr. J. Phys., 23, 347

Kilkenny, D., Marang, F., \& Menzies, J. W. 1994, MNRAS, 267, 535

Kilkenny, D., Keuris, S., \& Marang, F. 2000, The Observatory, 120, 48

Kilkenny, D., van Wyk, F., \& Marang, F. 2003, The Observatory, 123, 31

Kiss, L. L., Csak, B., Szatmary, K., Füresz, G., \& Sziladi, K. 2000, A\&A, 364, 199

Kwee, K. K., \& van Woerden, H. 1956, BAN, 12, 327 
Kirkpatric, J. D., Reid, I. N., \& Liebert, J. 2000, AJ, 120, 447

Maxted, P. F. L., Marsh, T. R., Heber, U., et al. 2002, MNRAS, 333, 231

Menzies, J. W. 1986, Ann. Rep. S. Afr. Astron. Obs., 1985, 20

Menzies, J. W., \& Marang, F. 1986, IAUS, 138, 305

Saffer, R. A., Green, E. M., \& Bowers, T. P. 2001, The Binary Origins of Hot Subdwarfs: New Radial Velocities, in Proc. of the 12th European Workshop on White Dwarf Stars, ed. H. L. Shipman, J. L. Provencal, J. MacDonald, \& S. Goodchild, ASP Conf. Ser., 226, 408
Wilson, R. E., \& Devinney, E. J. 1971, ApJ, 166, 605

Wilson, R. E. 1998, Computing Binary Star Observables, Reference manuel to the Wilson-Devinney Program, Department of Astronomy, University of Florida, Gainesville, FL

Wlodarczyk, K., \& Olszewski, P. 1994, Acta. Astron., 44, 407

Wood, J. H., Zhang, E.-H., \& Robinson, E. L. 1993, MNRAS, 261, 103

Wood, J. H., \& Saffer, R. 1999, MNRAS, 305, 820 